\title{
高镍系三元层状氧化物正极材料容量衰减机理的研究进展
}

\author{
李 想 ${ }^{1,2}$, 葛武杰 ${ }^{1,2}$, 王 昊 ${ }^{1,2}$, 㲘美臻 ${ }^{1}$
}

(1. 中国科学院 成都有机化学研究所, 成都 610041; 2. 中国科学院研究生院, 北京 100039)

摘 要: 高镍系三元层状氧化物正极材料因其高比容量、低廉的价格以及较好的环境友好性而受到广泛关注, 但是 其固有的一些缺点，如循环过程中结构稳定性差、高温稳定性差以及储存性能差等极大地限制了其在各领域的广泛 应用。本文着重总结并讨论近年来对高镍系三元层状氧化物正极材料循环过程容量衰减机理的研究进展，并对高镍 系三元层状氧化物正极材料的进一步改性作了简要的展望。

关 键 词: 高镍系氧化物; 三元层状氧化物; 正极材料; 衰减机理; 循环稳定性; 综述 中图分类号: TM912 文献标识码: A

\section{Research Progress on the Capacity Fading Mechanisms of High-Nickel Ternary Layered Oxide Cathode Materials}

\author{
LI Xiang ${ }^{1,2}$, GE Wu-Jie ${ }^{1,2}$, WANG Hao ${ }^{1,2}$, QU Mei-Zhen ${ }^{1}$
}

\begin{abstract}
(1. Chengdu Institute of Organic Chemistry, Chinese Academy of Sciences, Chengdu 610041, China; 2. Graduate University of Chinese Academy of Sciences, Beijing 100039, China)
\end{abstract}

\begin{abstract}
Owing to advantages of high specific capacity, low cost and environmental friendliness, high-nickel ternary layered oxide cathode materials have received much attention and have been extensively studied over the past ten years. However, its further application is hindered by its structural instability during the cycling, thermal instability and poor storage properties. In order to provide references to tackle the problems that high nickel ternary layered oxide cathode materials faces, the degradation mechanisms of high-nickel ternary layered oxide cathode materials during cycling was summarized and discussed in this review. The degradation mechanisms are categorized as two major factors. One is thermodynamic factors including cation disorder as well as surface reactions, and the other is kinetic factors comprising of micro-cracks formation as well as rearrangement of conductive materials. The modification strategies corresponding to the major mechanisms and the research focusing on initial irreversible capacity loss are also briefly introduced.
\end{abstract}

Key words: high-nickel oxide; ternary layered oxide; cathode material; degradation mechanisms; cycling stability; review

高镍系三元层状氧化物正极材料由于具有高比 容量、价格低廉和较好的环境友好性等优点而受到 广泛关注, 但是其较差的循环稳定性、热稳定性和储 存性能极大地限制了其应用 ${ }^{[1]}$ 。这些缺点大多是高镍 系三元层状氧化物正极材料的本质属性, 为克服它
们带来了较大的挑战 ${ }^{[2]}$, 近年来, 研究者们尝试了多 种方法，试图兼得高比容量和高稳定性两方面的优 点。常用的方法有掺杂 ${ }^{[3-6]}$ 、包覆 ${ }^{[7-11]}$ 以及制备成特殊 结构的材料 ${ }^{[12-13]}$, 然而这些工作对材料电化学性能的 改善程度并不理想。探寻一种可控的和重复性好的改 
性方法成为当务之急。因此, 从高镍系三元层状氧化 物正极材料容量衰减机理出发进行深入的研究, 并 找到造成其容量衰减的主、次要因素显得尤为关键, 已经成为近年来研究的热点。本文将对高镍系三元层 状氧化物正极材料容量衰减机理的研究做初步总结。

本文将高镍系层状氧化物正极材料定义为: 在 三元层状氧化物正极材料 $\mathrm{LiMO}_{2}$ 中, 金属元素 $\mathrm{M}$ 所 包含 $\mathrm{Ni}$ 元素的摩尔分数为 0.6 及以上的三元材料。 作为 $\mathrm{LiNiO}_{2}$ 的衍生物, 高镍系三元层状氧化物正极 材料均具有 $\alpha-\mathrm{NaFeO}_{2}$ 型层状结构, 属于 $\mathrm{R} \overline{3} \mathrm{~m}$ 空间 群。虽然通过用其他金属元素(Co、Mn、 $\mathrm{Al} 、 \mathrm{Ti}$ 等) 取代部分 $\mathrm{Ni}$ 元素形成多元固溶体氧化物能够大大 降低其非化学计量比, 稳定层状结构, 提升电化学 性能, 但是其仍在一定程度上保留了 $\mathrm{LiNiO}_{2}$ 的诸多 缺点 ${ }^{[14]}$, 首先是合成上的问题: (1)高温合成条件下, 锂盐容易挥发而导致锂缺陷产生; (2)从 $\mathrm{Ni}^{2+}$ 氧化到 $\mathrm{Ni}^{3+}$ 存在较大的势垒, 其氧化难以完全; (3)高温下, 其容易发生相变和分解反应, 会产生无化学活性的 立方岩盐相。其次是充放电过程中存在的问题: (1) 由于合成产物不可能完全符合化学计量比, 部分 $\mathrm{Ni}$ 占据锂层, 在充电过程中 $\mathrm{Ni}^{2+}$ 氧化成为 $\mathrm{Ni}^{3+}$ 或 $\mathrm{Ni}^{4+}$, 会造成间晶层(Interslab space)空间的局部塌陷, 增 加 $\mathrm{Li}^{+}$嵌入的阻力, 造成阻抗增大, 循环性能恶化; (2)随着锂离子的嵌入脱出, 高镍系层状氧化物正极 材料会发生一系列相变, 主要发生从六方相 $\mathrm{H} 1$ 到 单斜相 $\mathrm{M}$ 、到六方相 $\mathrm{H} 2$ 、到六方相 $\mathrm{H} 3$ 的一系列相 变。其中 $\mathrm{H} 1 \rightarrow \mathrm{M} \rightarrow \mathrm{H} 2$ 的相变是可逆的, 对电化学 性能的影响不大, 而 $\mathrm{H} 2 \rightarrow \mathrm{H} 3$ 的相变是不可逆的, 对电化学性能的影响很大。

为了方便讨论, 以下常见的正极材料将使用公 认的简写, 例如 $\mathrm{LiNi}_{0.8} \mathrm{Co}_{0.15} \mathrm{Al}_{0.05} \mathrm{O}_{2}$ 简写为 $\mathrm{NCA}$, $\mathrm{LiNi}_{0.8} \mathrm{Co}_{0.1} \mathrm{Mn}_{0.1} \mathrm{O}_{2}$ 简写为 $\mathrm{NCM} 811, \mathrm{LiNi}_{0.6} \mathrm{Co}_{0.2} \mathrm{Mn}_{0.2} \mathrm{O}_{2}$ 则简写为 NCM622 等等。另外, 由于高镍系层状氧 化物正极材料在较高的环境温度下容量衰减更加剧 烈, 研究的侧重点和常温下也有所不同, 所以本文 将分别论述。再者, 容量的衰减包含两个过程, 一是 首次充放电过程中损失的容量; 二是在循环过程中 的容量衰减。由于这两者在机理上有所差异, 并且 在首次充放电过程中的容量衰减属于材料的本质属 性, 难以克服, 所以仅在文末对其代表性的工作做 简要介绍。

\section{1 室温容量衰减机理}

首先, 本文对循环过程中高镍系层状氧化物正
极材料容量衰减的机理研究做较为详细的总结和分 析。循环过程中存在的容量衰减因素主要有阳离子 混排、应力诱导微裂纹的产生、固体-电解质界面膜 (SEI layer)的生成、导电炭黑的重新分布以及 HF 的 腐蚀作用等，其中以阳离子混排和应力诱导微裂纹 的产生两个因素对容量衰减的作用最为显著。它们 之间的作用很可能不是孤立存在的，而是具有交互 作用的, 这造成容量衰减速率逐渐增大。此外, 还可 以将容量衰减按热力学和动力学因素分为两大类, 其中阳离子混排、界面反应等可以归结为热力学因 素, 而微裂纹的产生、导电炭黑的重新分布等则可 以归结为动力学因素。

\section{1 热力学因素}

\subsection{1阳离子混排}

一般认为, 相变是由于高镍系层状氧化物正极 材料中阳离子混排(Cation disorder / Cation mixing) 造成的。根据晶体场理论, $\mathrm{Ni}^{3+}$ 是热力学不稳定的, 具有自发还原为 $\mathrm{Ni}^{2+}$ 的趋势。与此同时, $\mathrm{Ni}^{2+}$ 半径 (6.9 nm) 和 $\mathrm{Li}^{+}$半径 $\left(7.4 \mathrm{~nm}\right.$ ) 接近, 在循环过程中 $\mathrm{Li}^{+}$ 大量脱嵌形成锂位空缺, 而两个相邻的氧层之间产 生排斥作用, 这就给 $\mathrm{Ni}^{2+}$ 迁移到 $\mathrm{Li}$ 位提供了驱动力, 造成阳离子混排。为了稳定层状结构, $\mathrm{Ni}^{2+}$ 不断地占 据 $\mathrm{Li}$ 位，当 $\mathrm{Ni}^{2+}$ 完全占据了 $\mathrm{Li}$ 位，正极材料的空间 群结构就由 $\mathrm{R} \overline{3} \mathrm{~m}$ 转变为了 $\mathrm{Fm} \overline{3} \mathrm{~m}$ (立方岩盐相结构, 类 $\mathrm{NiO}$ 相)。虽然 $\mathrm{Co} 、 \mathrm{Mn}$ 元素也可能与 $\mathrm{Li}$ 发生阳 离子混排，但是其概率相对 $\mathrm{Ni} / \mathrm{Li}$ 混排太小，一般忽 略不计。研究发现, 上述反应的部位主要发生在正 极材料晶粒表层, 由于锂离子的传输速率在颗粒内 部各部位不相同，所以在充放电过程中会逐渐建立 锂离子浓度梯度, 并且缺锂的程度由表及里逐渐减 小, 表层晶格的扭曲程度最大, 发生相变反应的概 率也最大, 这就是所谓的表面重构 ${ }^{[15]}$, 这种表面重 构现象和材料中 $\mathrm{Ni}$ 元素的含量有密切关系。

研究验证, 对于 NCA 材料而言, 即便是未循环 过的原始材料, 只要暴露在空气中, 其表面也存在 一层类 $\mathrm{NiO}$ 相, 并且和电解液接触的一瞬间就会发 生反应生成更多类 $\mathrm{NiO}$ 相, 如图 1 所示 ${ }^{[16]}$ 。而对 于 $\mathrm{NCM}$ 材料, 由于 $\mathrm{Mn}$ 元素以 +4 价的形式存在, 为 了保持电荷平衡, 必然存在一部分 $\mathrm{Ni}$ 以 +2 价形式 存在，在合成过程中表层会出现一层 $\mathrm{NiO}$ 相 ${ }^{[17]}$ 。

$\mathrm{Ni}^{2+}$ 的不断产生造成 $\mathrm{Ni} / \mathrm{Li}$ 混排程度增大并逐 渐产生 $\mathrm{Li}^{+}$传导性差的类 $\mathrm{NiO}$ 相, 研究者普遍认为 在高镍系层状氧化物正极材料当中, 应当尽量避免 $\mathrm{Ni}^{2+}$ 的产生。然而研究发现, 对于 $\mathrm{NCM}$ 材料而言, 在表面形成一层类 $\mathrm{NiO}$ 相有助于提升材料的循环稳 


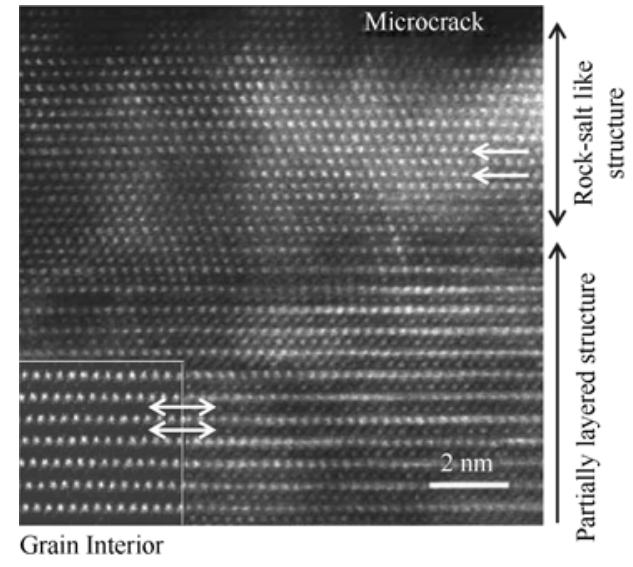

图 1 循环 10 次以后 NCA 晶界处的高分辨率扫描透射电镜 图像 ${ }^{[16]}$

Fig. 1 High-resolution STEM image of $\mathrm{LiNi}_{0.8} \mathrm{Co}_{0.15} \mathrm{Al}_{0.05} \mathrm{O}_{2}$ at a grain boundary layer after ten cycles ${ }^{[16]}$

定性。Cho 等 ${ }^{[17-18]}$ 在 $\mathrm{LiNi}_{0.7} \mathrm{Co}_{0.15} \mathrm{Mn}_{0.15} \mathrm{O}_{2}$ 材料表面 掺入 $\mathrm{Mn}$ 元素, 材料的表面层中具有更高的 $\mathrm{Mn}^{4+}$ 含 量, 由于电荷补偿作用, 一部分 $\mathrm{Ni}^{3+}$ 还原为 $\mathrm{Ni}^{2+}$ 。与 此同时, 部分 $\mathrm{Ni}^{2+}$ 趋向于迁移至 $\mathrm{Li}$ 位(造成较大的 阳离子混排), 位于 $\mathrm{Li}$ 层的少量 $\mathrm{Ni}^{2+}$ 能起到稳定或 者支撑 $\mathrm{Li}$ 层的作用, 称之为 “支撑效应”，它能有 效抑制内核材料的进一步相变, 减弱内核的应力应 变效应，从而提高循环稳定性，如图 2 所示。

研究者发现在高镍系层状氧化物材料中掺杂如 $\mathrm{Mg}^{2+[5,19-20]}$ 等与 $\mathrm{Li}^{+}$半径相近的离子, 能够有效改善 其循环稳定性。 $\mathrm{Mg}^{2+}$ 能优先占据 $\mathrm{Li}$ 位, 一方面抑制 镍锂混排, 一方面可以屏蔽 $\mathrm{O}^{2-}-\mathrm{O}^{2-}$ 的排斥作用, 从 而防止层状结构塌陷, 提高结构稳定性。对于 NCA 材 料, 掺入 $\mathrm{Mg}$ 可以有效抑制 $\mathrm{H} 2-\mathrm{H} 3$ 的相变反应, 提高 其在高充放电截止电势下的循环稳定性 ${ }^{[21]}$ 。 $\mathrm{Mg}^{2+}$ 的作 用类似于支撑效应, 如图 3 所示, 相比 $\mathrm{Ni}^{2+}$, 它在充 放电过程中表现为惰性, 既无价态变化, 亦无尺寸 变化, 能起到支撑 $\mathrm{Li}$ 层的作用, 保证回嵌通道不被 阻碍, 从而提高循环稳定性 ${ }^{[22]}$ 。但它的副作用则是 首次充放电效率下降, 放电容量降低较为明显。

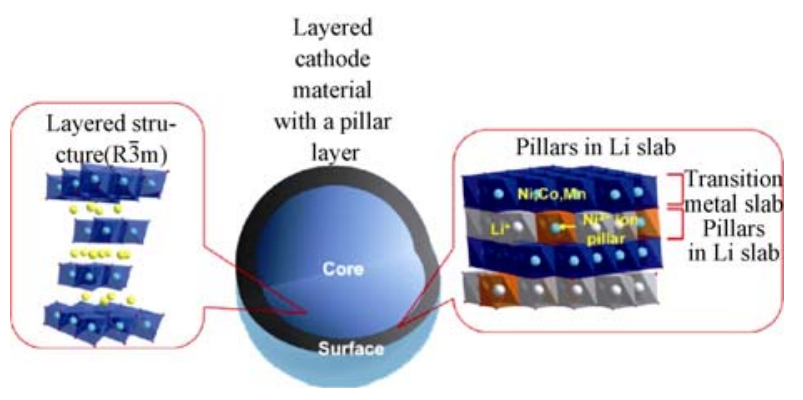

图 2 正极材料表面支柱层示意图 ${ }^{[17]}$

Fig. 2 Schematic view of the layered cathode material with a pillar layer at the surface ${ }^{[17]}$

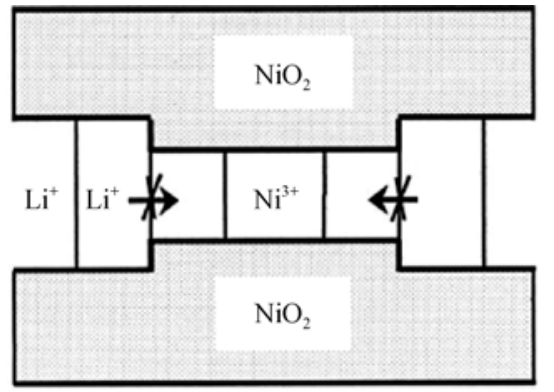

(a)

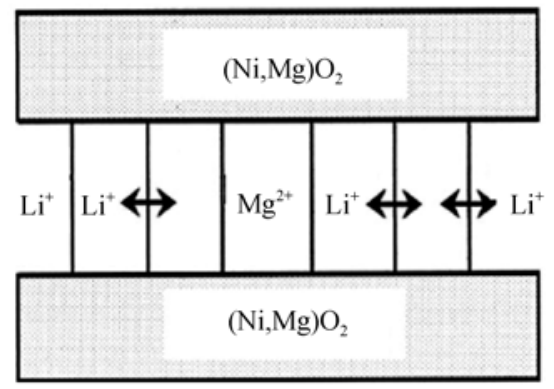

(b)

图 $3 \mathrm{Li}$ 层空间示意图 ${ }^{[22]}$

Fig. 3 Schematic representation of the inter-slab space ${ }^{[22]}$ (a) In the $\mathrm{Li}_{x} \mathrm{Ni}_{1+z} \mathrm{O}_{2}$ system, the oxidation of the $\mathrm{Ni}^{2+}$ ions during the cycling induces a local collapse of the inter-slab space which makes lithium diffusion and re-intercalation difficult; (b) The $\mathrm{Li}_{x} \mathrm{Ni}_{1-y} \mathrm{MgO}_{2}$ system, the electrochemically inactive $\mathrm{Mg}^{2+}$ ions do not hinder lithium diffusion since their size is very close to that of the $\mathrm{Li}^{+}$ion

$\mathrm{Wu}$ 等 ${ }^{[23]}$ 对 NCM811 材料进行不同混锂量的研 究, 发现随着混锂量从 $100 \%$ 依次增加 10\%直到 $130 \%$, 材料的放电容量尽管有所下降，但循环性能却发生 了明显的改善。XPS、XRD 数据进一步表明, 由于 电荷平衡的要求, 过多的 $\mathrm{Li}^{+}$导致部分 $\mathrm{Ni}^{3+}$ 还原为 $\mathrm{Ni}^{2+}$ ，存在适量 $\mathrm{Ni}^{2+}$ 反而减小了整体材料 $\mathrm{Li} / \mathrm{Ni}$ 混排 的程度。陈涨宗等 ${ }^{[24]}$ 通过富锂的方式在空气气氛中 制备 NCA 材料, 研究发现过量 $\mathrm{LiOH}$ 能替代 $\mathrm{O}_{2}$ 抑 制烧结过程中的 $\mathrm{Ni} / \mathrm{Li}$ 混排, 结果表明: 当锂源/前 驱体摩尔比为 1.15 时, 在氧气和空气中合成的 NCA 材料的形貌、结构和电化学性能相当。

阳离子的混排通常只被归因于 $\mathrm{Ni}^{2+} 、 \mathrm{Li}^{+}$的离子 半径相近, 然而 $\mathrm{Bi}$ 等 ${ }^{[25]}$ 对 NCM811 材料研究发现, 阳离子混排可能与氧空位的存在有着密切的联系。 通过对 XRD 数据进行结构精修分析发现, NCM811 材料 $6 \mathrm{c}$ 位的氧占位量随着烧结过程中氧分压的升 高而提高; 相反, 氧分压越低氧空位越多。进一步的 分析表明, 由于氧空位的存在可能会降低 $\mathrm{Ni} / \mathrm{Li}$ 混 排的转变势垒, 从而诱发氧空位处 $\mathrm{Ni} / \mathrm{Li}$ 混排, 相变 反应将优先在氧空位处发生, 此空位也可能是真正 的催化活性中心(相比于 $\mathrm{Ni}^{3+}$ )。所以低氧分压条件 下制备的材料首次放电容量都较低, 循环性能较 差。进一步研究发现氧分压越低, 一次颗粒表面的 
杂相层(包括类 $\mathrm{NiO}$ 相和 $\mathrm{Li}_{2} \mathrm{CO}_{3}$ )越厚, 这可能是由 于失氧过程中伴随着 $\mathrm{Li}$ 的析出和类 $\mathrm{NiO}$ 相的生成, $\mathrm{Li}$ 与空气中 $\mathrm{CO}_{2}$ 结合转变为 $\mathrm{Li}_{2} \mathrm{CO}_{3}$, 如下式所示。

$4 \mathrm{LiNiO}_{2-x}+2 \mathrm{CO}_{2} \rightarrow 4 \mathrm{NiO}+2 \mathrm{Li}_{2} \mathrm{CO}_{3}+(1-2 x) \mathrm{O}_{2}$

以上研究在一定程度上可以解释低镍正极材料 $\mathrm{Ni}^{2+}$ 的含量更高 (例如 $\mathrm{LiNi}_{1 / 3} \mathrm{Co}_{1 / 3} \mathrm{Mn}_{1 / 3} \mathrm{O}_{2}$ 和 $\mathrm{LiNi}_{0.4} \mathrm{Co}_{0.2} \mathrm{Mn}_{0.4} \mathrm{O}_{2}$ 等材料)却能表现出优良的循环 稳定性, 这可能与其具有较低的氧空位有关, 如图 4 所示。 $\mathrm{F}$ 的电负性比 $\mathrm{O}$ 强, 很难被氧化, 如果使用 $\mathrm{F}$ 部分替换 $\mathrm{O}$ 占据 $6 \mathrm{c}$ 位可能会起到稳定氧层, 减 小氧层之间静电斥力, 并提高材料循环稳定性的 作用 ${ }^{[26]}$ 。

\subsection{2 界面反应}

Zhuang 等 ${ }^{[27]}$ 将 NCA 材料暴露在空气中一段时 间, 发现表面生成一层较厚的膜层, 通过红外和 XRD 等手段证实其为 $\mathrm{Li}_{2} \mathrm{CO}_{3}$, 它可能是 NCA 与 $\mathrm{H}_{2} \mathrm{O} / \mathrm{CO}_{2}$ 的反应产物。使用小电流循环后 XRD 图谱 显示(003)峰为双峰, 意味着存在两个相态, 一个是 发生了嵌脱锂反应的相, 另一个则与原始相态相同, 原因可能是 $\mathrm{Li}_{2} \mathrm{CO}_{3}$ 的包裹使得部分 NCA 颗粒被隔 离, 这部分活性材料无法进行充放电, 所以表现出 与其他部分不同的充电状态, 也同时表现出不同的 相结构。通过表面包覆 $\mathrm{LiCoO}_{2}$ 或 $\mathrm{NH}_{4} \mathrm{~F}$ 改性处理后 能够有效提升 NCA 在空气中抵抗 $\mathrm{CO}_{2}$ 和 $\mathrm{H}_{2} \mathrm{O}$ 的能

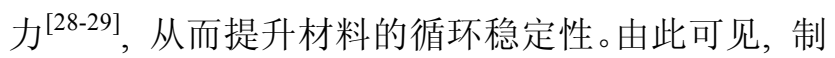
备环境对高镍系层状氧化物正极材料的性能有较大 的影响, 制备条件要求比较苛刻。

Xiong 等 ${ }^{[30]}$ 对 NCM811 材料进行水洗并研究其 储存和电化学性能, 结果表明水洗能有效地减少材 料表面的残余碱, 但是水洗后的材料在空气中抵抗 $\mathrm{H}_{2} \mathrm{O} / \mathrm{CO}_{2}$ 的能力下降, 在空气中存放 $30 \mathrm{~d}$ 后, 其表 面的 $\mathrm{LiOH} / \mathrm{Li}_{2} \mathrm{CO}_{3}$ 含量是未水洗材料的 2 倍, 并且 阳离子混排程度更高。进一步研究指出, 在对材料 进行湿法包覆处理的过程中, 材料表面未被包覆的

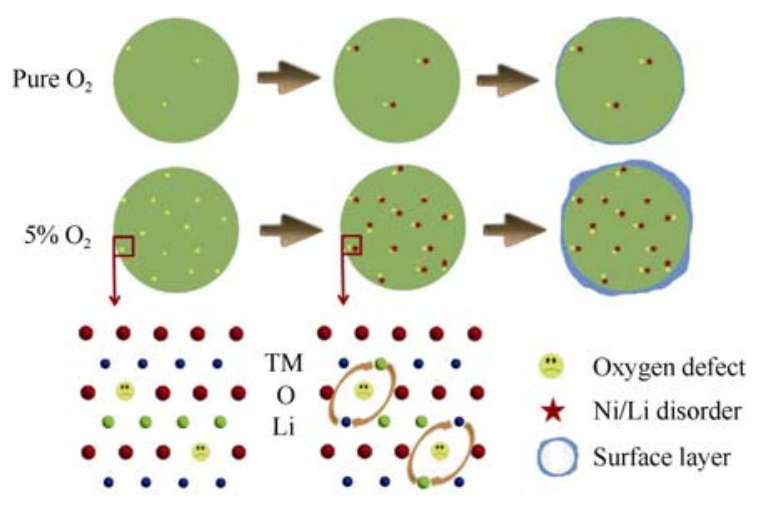

图 4 氧缺陷对结构不稳定性的影响 ${ }^{[25]}$

Fig. 4 Influence of oxygen defect on structural instabilities ${ }^{[25]}$
裸露区域类似水洗后的材料，如果这些区域过多， 则材料的循环性能可能会恶化。因此，包覆的均匀 性尤为重要。Zheng 等 ${ }^{[31]}$ 发现乙醇洗涤 NCM811 材 料后, $\mathrm{Li}_{2} \mathrm{CO}_{3}$ 含量明显比未洗涤材料少, 并且在后 续储存过程中，抑制了洗涤材料表面产生 $\mathrm{Li}_{2} \mathrm{CO}_{3}$, 使得材料对 $\mathrm{H}_{2} \mathrm{O} / \mathrm{CO}_{2}$ 的抵抗能力变强, 并减少了 $\mathrm{Ni}^{3+}$ 转变为 $\mathrm{Ni}^{2+}$ 的程度, 提高了材料的循环稳定性, 因此乙醇洗涤比水洗的效果好。

$\mathrm{Bi}$ 等 ${ }^{[32]}$ 为研究 $\mathrm{Li}_{2} \mathrm{CO}_{3}$ 的化学稳定性, 将其与 含 $\mathrm{LiPF}_{6}$ 的电解液混合后封装置于铝塑膜中, 并在 不同温度下放置一定时间，研究发现生成的固态物 质为 $\mathrm{LiF}$, 产生的气体 $90 \%$ 以上为 $\mathrm{CO}_{2}$ 和 $\mathrm{POF}_{3}$, 其 中 $\mathrm{CO}_{2}$ 来自 $\mathrm{Li}_{2} \mathrm{CO}_{3}, \mathrm{POF}_{3}$ 来自 $\mathrm{PF}_{6}{ }^{-}$与痕量的水的反 应。进一步研究发现固态 $\mathrm{Li}_{2} \mathrm{CO}_{3}$ 和 $\mathrm{LiPF}_{6}$ 混合产生 的反应与 $\mathrm{Li}_{2} \mathrm{CO}_{3}$ 和电解液混合相同。结果表明, $\mathrm{Li}_{2} \mathrm{CO}_{3}$ 的分解可能不需要水的参与, 如下式所示。

反应路径 1 :

$$
\mathrm{LiPF}_{6}+\mathrm{Li}_{2} \mathrm{CO}_{3} \rightarrow \mathrm{POF}_{3}+\mathrm{CO}_{2}+3 \mathrm{LiF}
$$

同时, 他们发现将 $\mathrm{LiPF}_{6}$ 加入大量水中无沉淀, $\mathrm{pH}$ 为 5; 而将水缓慢加入 $\mathrm{LiPF}_{6}$ 中产生 $\mathrm{LiF}$, 并且 $\mathrm{pH}$ 为 2 。可见 $\mathrm{LiPF}_{6}$ 趋向于和少量的水发生分解反应产 生 $\mathrm{H}^{+}, \mathrm{PF}_{6}{ }^{-}$基团难以和少量水形成稳定的水化层, 反应如下。

反应路径 2 :

$$
2 \mathrm{H}^{+}+\mathrm{Li}_{2} \mathrm{CO}_{3} \rightarrow 2 \mathrm{Li}^{+}+\mathrm{CO}_{2}+\mathrm{H}_{2} \mathrm{O}
$$

$\mathrm{LiPF}_{6}+2 \mathrm{Li}^{+}+\mathrm{H}_{2} \mathrm{O} \rightarrow \mathrm{POF}_{3}+2 \mathrm{H}^{+}+3 \mathrm{LiF}$

以上是 $\mathrm{Li}_{2} \mathrm{CO}_{3}$ 分解的两条可能路径, 研究者认 为反应路径 1 的可能性较大。他们将 NCM 811 材料 置于 $\mathrm{H}_{2} \mathrm{O} / \mathrm{CO}_{2}$ 环境中生成了 $\mathrm{Li}_{2} \mathrm{CO}_{3}$ 包覆 $\mathrm{NCM} 811$, 将其与电解液混合置于 $80^{\circ} \mathrm{C}$ 下 $12 \mathrm{~h}$ 则生成 $\mathrm{LiF}$ 包覆 $\mathrm{NCM} 811$, 后者的循环性能比前者以及原始 NCM811 更好。可见当 NCM811 材料表面存在较多 的 $\mathrm{Li}_{2} \mathrm{CO}_{3}$, 在循环过程中分解产生气体, 吸附于材 料的表面造成活性物质与电解液的接触不佳, 极化 增大, 循环性能也随之恶化。

\section{2 动力学因素}

\subsection{1 微裂纹的产生}

晶粒内部应力变化诱导产生微裂纹是另一个影 响容量衰减非常重要的因素 ${ }^{[33]}$ 。从微观上来看, 锂 离子脱嵌伴随着晶胞参数的变化 ${ }^{[34]}$; 从宏观上来看, 放电时材料膨胀, 充电时收缩 ${ }^{[35-36]}$ 。微裂纹的产生 实质上是相结构变化的衍生物。高镍系层状氧化物 正极材料二次颗粒由取向各不相同的多晶组成，晶 粒之间存在着许多微空隙。在循环过程中, 发生各 向异性晶格膨胀和收缩, 导致晶界空隙处产生裂纹, 并逐渐延伸。长时间或极端条件下循环会造成正极 
材料球形颗粒粉化或形成中空结构。不仅一次颗粒 之间产生裂纹, 不同方向的膨胀同样可在一次颗粒 内部产生裂纹。由于裂纹的产生，一方面一次颗粒 之间的接触变差甚至有的一次颗粒完全被孤立, 这 部分活性物质参与电化学反应的能力必然减弱, 另 一方面电解液的浸入会形成新的 SEI 膜, 导致阻抗 升高 ${ }^{[37-38]}$ 。虽然电解液的浸润利于锂离子的脱嵌, 但是缺乏与导电物质的接触, 被孤立的颗粒也难以 提供容量。随着循环的进行, 被孤立的一次颗粒增 多, 整体材料内部充放电速率不均匀, 进一步恶化 材料性能, 如图 5 所示 ${ }^{[16]}$ 。体积变化的程度与镍含 量的多少有关, NCM811 材料在充放电过程中会产 生 $9 \% \sim 10 \%$ 的体积变化, 而 $\mathrm{LiNi}_{0.5} \mathrm{Mn}_{0.5} \mathrm{O}_{2}$ 在嵌脱锂 的过程中则仅仅膨胀收缩 $3 \%$ 。裂纹的产生还依赖 充放电截止电势的大小, 所以通常高镍系层状氧化 物正极的工作电压(相对于锂金属负极)不超过 $4.1 \mathrm{~V}$, 目的是为了保证不发生不可逆相变, 减小内应力。 当充电电势高于 $4.2 \mathrm{~V}$, 将发生 $\mathrm{H} 2 \rightarrow \mathrm{H} 3$ 的不可逆相 变, 造成较大的体积变化, 产生许多 $\mathrm{NiO}_{2}$ 微区, 这 既诱导材料进一步发生相变, 又会引发材料产生裂 纹 ${ }^{[39-42]}$ 。如果能减弱在此电势范围内的相变反应, 就有希望在保证材料的结构稳定的前提下, 尽可能 大地拓宽其工作电压以提高放电容量。

前文提及 $\mathrm{Mg}^{2+}$ 掺杂是一种较为有效的抑制相 变反应, 减弱体积膨胀效应的方法。研究发现, 使用 改进的共沉淀法制备的浓度梯度正极材料中, 晶粒 呈条状或棒状, 并且晶胞的 $c$ 轴基本垂直于棒的轴 向平面, 这大大减小了锂离子传导过程中的晶界数 量, 极大便利了锂离子的传导, 如图 6 所示 ${ }^{[43]}$ 。这 种微观形貌使得材料在高充电截止电势下, 膨胀的 方向大体一致, 颗粒内部的各向异性应力变为局部 的各向同性, 极大减少微裂纹的产生, 循环性能较 普通材料优异。Kim 等 ${ }^{[18]}$ 研究发现通过在 NCM622
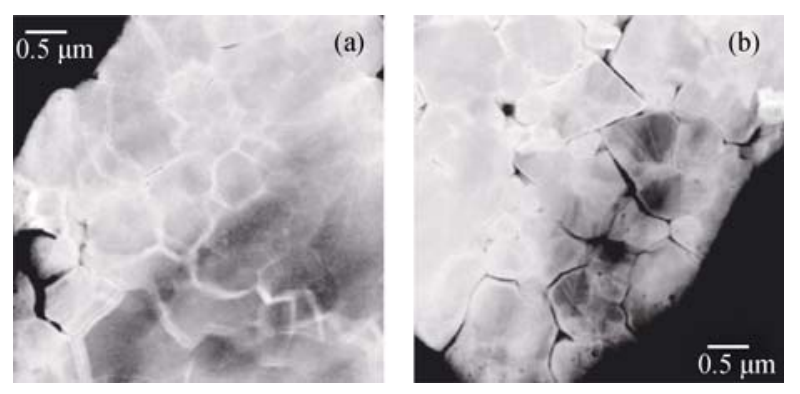

图 $5 \mathrm{NCA}$ 在(a)首次循环和(b)10 次循环后的扫描透射电镜 图像 ${ }^{[16]}$

Fig. 5 STEM images of $\mathrm{LiNi}_{0.8} \mathrm{Co}_{0.15} \mathrm{Al}_{0.05} \mathrm{O}_{2}$ after (a) first cycle and (b) ten-cycles ${ }^{[16]}$
材料混入硝酸钴并烧结在其晶界处形成 $\mathrm{Fm} \overline{3} \mathrm{~m}$ 相, 包裹住内部的 $\mathrm{R} \overline{3} \mathrm{~m}$ 相。从高分辨率透射电镜图中可 以明显看出表面处理过的材料晶界处的阳离子混排 层( 10 nm)远厚于未处理过的材料(1 2 nm)。进一步 研究循环后材料的横截面透射电镜图发现, 表面未 处理的材料二次颗粒之间缝隙增大明显, 并且在其 一次颗粒内部也发现了大量的裂缝, 而表面处理后 的材料则未产生大裂缝, 循环性能得到明显提升。 Yang 等 ${ }^{[44]}$ 通过共沉淀法将 NCM811 材料制备成内 部均匀嵌入 $\mathrm{Li}_{2} \mathrm{MnO}_{3}$ 结构单元的两相复合材料, 可 以有效抑制 H2-H3 相变反应的发生, 同时减弱体积 的变化, 在一定程度上提高了材料在高电压下的循 环稳定性。

\subsection{2 导电物质的重新分布}

Kostecki 等 ${ }^{[45-48]}$ 采用原位 Raman 光谱和原子力 显微镜详细研究了 NCA 材料表面的导电物质在循 环前后的分布状况。通过 Raman 半定量研究发现导 电物质在循环后存在重新分布或者离开活性物质表 面的现象, 通过原子力显微镜研究发现 NCA 颗粒 表面的导电性由于导电物质的脱离而大大降低, 如 图 7 所示。这一现象造成材料的电荷传递阻抗增大, 并且由于导电物质的分布发生变化导致部分区域导 电性好，而另外部分区域则处于导电不良的状态。 通过拉曼特征峰的比值发现表面上晶格中 $\mathrm{Li}^{+}$的含 量不均匀, 即是材料颗粒表面或者不同颗粒间的充 电状态和充电电流密度呈现不均匀化。这种不均匀 的动力学行为使部分区域处于过充或过放状态, 导 致一系列不利反应的发生, 诸如电解液的分解、相 结构的不可逆变化以及颗粒之间的和颗粒内部的机
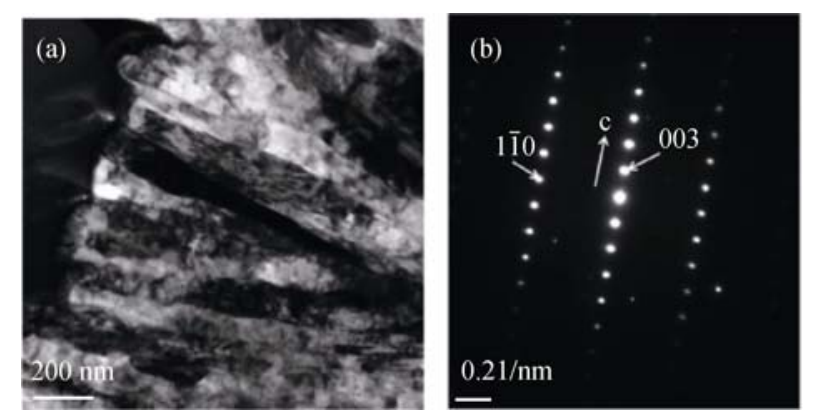

图 6 (a)经过锂化的正极颗粒界面透射电镜图显示单个一次 颗粒中成排的纳米结构, (b)单个纳米片的电子衍射图像显示 晶胞的 $\mathrm{c}$ 轴垂直于片的基本面 ${ }^{[43]}$

Fig. 6 (a) TEM image of cross-section of lithiated particle showing the aligned nanostructure inside a single particle, (b) Electron diffraction pattern on a single nanoplate showing that the $\mathrm{c}$ axis (in $\mathrm{R}^{-} \mathrm{m}$ space) is perpendicular to the basal plane of the plate ${ }^{[43]}$ 
(a) Topotraphy

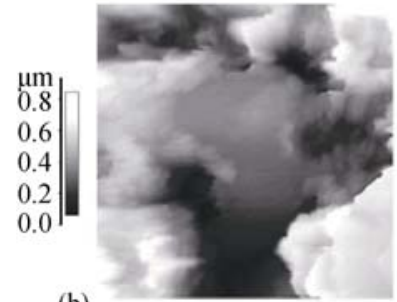

(b)
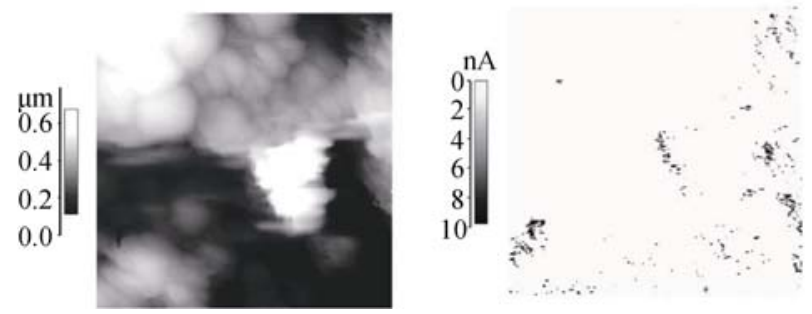

图 $75 \mu \mathrm{m} \times 5 \mu \mathrm{m}$ 区域内 $\mathrm{LiNi}_{0.8} \mathrm{Co}_{0.15} \mathrm{Al}_{0.05} \mathrm{O}_{2}$ 表面导电性(右边) 和形貌(左边)的电流响应原子力显微镜图 (接触电压 $1.0 \mathrm{~V})^{[45]}$ Fig. 7 CSAFM images of surface conductance (right-hand panel) and topography (left-hand panel) of a $5 \times 5 \mu \mathrm{m}$ region of the composite $\mathrm{LiNi}_{0.8} \mathrm{Co}_{0.15} \mathrm{Al}_{0.05} \mathrm{O}_{2}$ cathode surface at $1.0 \mathrm{~V}$ tip-sample voltage difference ${ }^{[45]}$

(a) Virgin cell; (b) Cathode from the cell which lost $34 \%$ of power

械碎裂等。如果导电物质能够形成一种致密均匀的 网络结构, 将一次颗粒与集流体紧紧连结起来, 那 么活性物质将得到充分的利用, 并且能够得到低阻 抗的电极材料。NCA 材料在循环后导电物质会发生 重新分布, 但是对于 $\mathrm{NCM}$ 材料, 这一方面的研究还 未见报道。

\section{2 高温容量衰减机理}

Sasaki 等 ${ }^{[49]}$ 使用 XRD、电子能量损失谱(EELS) 和 X 射线吸收精细结构谱(XANES)研究了 NCA 材 料在 3.0 4.1V 电压范围内, 不同温度下 $\left(20^{\circ} \mathrm{C} 、 60^{\circ} \mathrm{C}\right.$ 、 $70^{\circ} \mathrm{C} 、 80^{\circ} \mathrm{C}$ )循环 500 次后的性能变化。XRD 结果 显示, 循环前后材料的晶体结构没有明显的变化; EELS 和 XANES 的研究结果表明高温循环后材料 中存在非活性的 $\mathrm{Ni}$ 离子(包括二价和三价), 它在后 续充放电过程中不参与电荷补偿, 因而不能为材料 提供容量。将非活性 $\mathrm{Ni}$ 离子含量换算成不可逆容量 的结果表明, 计算所得的不可逆容量和实际不可逆 容量基本吻合，这在一定程度上说明在低电势窗口 内、高温条件下, 容量衰减主要是由于非活性 $\mathrm{Ni}$ 离 子的产生。进一步研究发现, 非活性 $\mathrm{Ni}^{2+}$ 主要来自 于集中在晶界和晶粒表面处的类 $\mathrm{NiO}$ 相，而一部分 非活性 $\mathrm{Ni}^{3+}$ 可能来自于缺锂相 $\mathrm{NiO}_{2}$, 而另一部分非 活性 $\mathrm{Ni}^{3+}$ 来源则尚不清楚。由于非活性 $\mathrm{Ni}$ 离子的存
在，在锂脱出的过程中可能会伴随着氧的逸出 ${ }^{[50]}$ 。

通过扫描透射电镜一电子能量损失谱法(STEMEELS) 研究发现, NCA 在 $70^{\circ} \mathrm{C}$ 下循环 500 次后生成 的类 $\mathrm{NiO}$ 相主要集中分布在材料颗粒的表面和晶界 处。通过定量计算发现类 $\mathrm{NiO}$ 相的形成是容量衰减 的主要原因。研究发现, 高温下脱锂的正极 $\mathrm{Ni}_{1-x}\left(\mathrm{Co}_{x}\right) \mathrm{O}_{2}$ 容易发生坍塌, 由 $\mathrm{NiO}_{6}$ 八面体蜕变为类 $\mathrm{NiO}$ 相, 所以提高 $\mathrm{Ni}-\mathrm{O}$ 键的结合强度很重要 ${ }^{[51]}$ 。

XRD 和质谱联用对 NCA 材料高温稳定性的研 究发现, 充电状态对材料结构的变化和气体的产生 都有较大影响，而气体的产生与相变反应又有着密 切的联系。充电截止电势越高, NCA 的结构稳定性 越差，失氧越严重。进一步使用 XANES 和 EXAFS 研究发现, 氧气的产生与过渡金属的迁移有密切关 系, 因为过渡金属的迁移直接影响着相变动力学, 如图 8 所示。另外，Ni 更容易直接形成岩盐相，而 Co 则趋向于先形成尖晶石相, 再过渡到岩盐相 ${ }^{[52]}$ 。

通过对过充的 NCA 材料进行原位 XRD 和原位 TEM 分析发现，室温下材料表层由内向外依次由六 方晶相、尖晶石相和岩盐相组成。当温度升高, 尖 晶石相向材料内部扩散, 同时, 岩盐相向尖晶石相 区域生长。温度升高到 $400^{\circ} \mathrm{C}$ 以上时, 大多数的材料 颗粒都转变成了岩盐相。另外, 过度脱锂的材料表 面发生氧流失，随之所形成的岩盐相成为相变产生 的晶核中心, 更加有利于尖晶石相向岩盐相的转变, 从而导致热稳定性急剧下降 ${ }^{[53]}$ 。另外, 通过 XRD、 拉曼光谱(Raman spectra)和电流响应原子力显微镜 (CSAFM)的研究发现，高温下容量的衰减与 SEI 膜 较差的锂离子传导性有关系 ${ }^{[54]}$ 。

高镍系层状氧化物正极材料在常温环境下存在 的结构变化和缺陷，在高温下表现得更加明显。随 着热运动加剧，相变的势垒更容易被克服，相变反 应更加剧烈, 氧的析出也变得更为严重。尤其是当

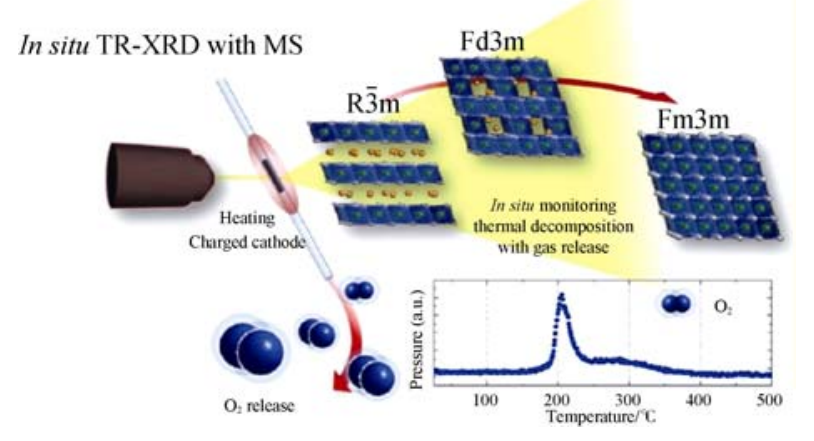

图 8 原位 XRD 和质谱联用分析结果示意图 ${ }^{[52]}$

Fig. 8 Schematic of the results from in situ TR-XRD with MS analysis $^{[52]}$ 
材料处于脱锂的介稳状态, 层状结构变得脆弱, 更 容易发生塌陷。所以, 高温下材料的放电容量更高, 但同时容量衰减也更为剧烈。

\section{3 活化过程容量衰减}

对 NCA 的前两次充放电循环过程进行原位 XRD 研究表明, NCA 在首次充电过程中将发生由 $\mathrm{R} 1$ 相到 R2 相的不可逆相变。此相变发生在 $0<x<0.5\left(\mathrm{Li}_{1-x} \mathrm{Ni}_{0.8} \mathrm{Co}_{0.15} \mathrm{Al}_{0.05} \mathrm{O}_{2}\right)$ 的组成范围内。其中, $\mathrm{R} 1$ 相离子传导性能差, R2 相较好, 所以首次充电表 现出更高的电位平台。此相转变的程度主要依赖于 充电截止电压或充电状态(SOC), 当电压低于 $4.1 \mathrm{~V}$ 时, 后续循环过程表现出明显的容量升高后趋于稳 定的过程; 在电流足够小的情况下进行完全充电能 完成 R1 到 R2 的相变, 第二次循环则以固溶反应的方 式进行, 电位平台变化不大 ${ }^{[34]}$ 。所以, 对于 $\mathrm{NCA}$ 材料 而言, 首次充放电的不可逆容量损失主要来自于 R1 到 R2 的不可逆相变, 此相变的程度代表了 $\mathrm{NCA}$ 材料 的活化程度。对于 $\mathrm{NCM}$ 材料, 暂时未见相关报道。

\section{4 总结与展望}

作为最具有前景的高容量锂离子电池正极材料 之一, 高镍系层状氧化物正极材料得到了广泛关 注。但是, 其存在的不足给研究者带来了较大的挑 战, 其应用也存在较大的技术壁垒, 如图 9 所示。通 过对高镍系层状氧化物正极材料容量衰减机理的研 究发现, 影响其高镍系三元层状氧化物正极材料容 量衰减的因素很多, 并且错综复杂。高镍系层状氧 化物正极材料不论 NCA 材料还是 NCM 材料面临的 问题主要可以归纳为三个方面: 一是循环过程中的 $\mathrm{Ni} / \mathrm{Li}$ 混排, 产生相变反应, 进而诱发应力应变效应; 二是材料表面与空气和电解液容易发生副反应; 三 是高温条件下材料的结构稳定性差和表面催化活性 较大。目前为止, 较为有效的解决方法主要有两种: 一是元素掺杂, 如 $\mathrm{Mg}$ 和 $\mathrm{F}$ 等, 可以有效减弱循环过 程中和高温高充电截止电势下的相变反应; 二是表 面修饰, 一方面包覆的厚度需要足够大且均匀, 以 抑制表面的副反应从而保护活性物质, 另一方面表 面包覆层又需要尽可能的薄, 以保证 $\mathrm{Li}^{+}$的传导不 受阻碍。值得指出的是, 氧原子/离子在其中很可能 起到至关重要的作用, 如何稳定氧原子/离子或如何 调控氧缺陷是很值得研究的方向。当然, 这其中还 存在许多问题需要进行更加深入的研究。要想大幅 度地改善电池的性能, 不仅要稳定材料的晶格结

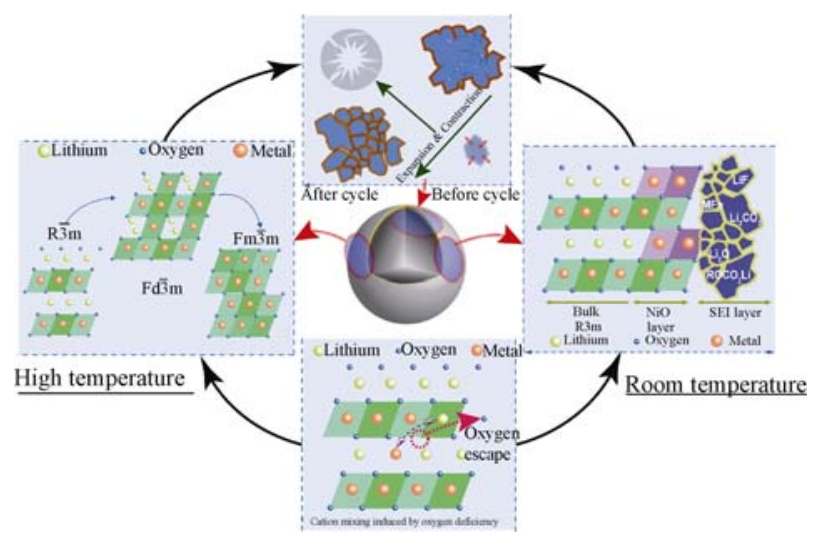

图 9 高镍系层状氧化物正极材料容量衰减机理示意图

Fig. 9 Schematic of capacity fading mechanism of high-nickel layered cathode materials

构、改善电极材料界面性能，还要综合考虑正极材 料和负极、电解液的匹配等问题。所以，高镍系层 状氧化物正极材料的广泛应用还需要不断探索。

致谢：感谢于作龙老师和彭工厂老师的指导和帮助。

\section{参考文献:}

[1] LIU W, OH P, LIU X E, Nickel-Rich layered lithium transition-metal oxide for high-energy lithium-ion batteries. Angewandte Chemie International Edition, 2015, 54: 4440-4457.

[2] YUAN RONG-ZHONG, QU MEI-ZHEN, YU ZUO-LONG. Thermal stability of nickel-based lithium transition metal oxides as the cathode materials for lithium-ion batteries. Journal of Inorganic Materials, 2003, 18(5): 973-979.

[3] LI X L, KANG F Y, SHEN W C, et al. Improvement of structural stability and electrochemical activity of a cathode material Li$\mathrm{Ni}_{0.7} \mathrm{Co}_{0.3} \mathrm{O}_{2}$ by chlorine doping. Electrochimica Acta, 2007, 53(4): 1761-1765.

[4] WOO S W, MYUNG S T, BANG H, et al. Improvement of electrochemical and thermal properties of $\mathrm{LiNi}_{0.8} \mathrm{Co}_{0.1} \mathrm{Mn}_{0.1} \mathrm{O}_{2}$ positive electrode materials by multiple metal $(\mathrm{Al}, \mathrm{Mg})$ substitution. Electrochimica Acta, 2009, 54(15): 3851-3856.

[5] MUTO S, TATSUMI K, KOJIMA Y, et al. Effect of Mg-doping on the degradation of $\mathrm{LiNiO}_{2}$-based cathode materials by combined spectroscopic methods. Journal of Power Sources, 2012, 205: 449-455.

[6] LIU W M, HU G R, DU K, et al. Surface coating of Li$\mathrm{Ni}_{0.8} \mathrm{Co}_{0.15} \mathrm{Al}_{0.05} \mathrm{O}_{2}$ with $\mathrm{LiCoO}_{2}$ by a molten salt method. Surface and Coating Technology, 2013, 216: 267-272.

[7] XIONG X H, WANG Z X, YAN G C, et al. Role of $\mathrm{V}_{2} \mathrm{O}_{5}$ coating on $\mathrm{LiNiO}_{2}$-based materials for lithium ion battery. Journal of Power Sources, 2014, 245: 183-193.

[8] HUANG B, LI X H, WANG Z X, et al. Enhanced electrochemical 
performance in $\mathrm{LiNi}_{0.8} \mathrm{Co}_{0.15} \mathrm{Al}_{0.05} \mathrm{O}_{2}$ cathode material: Resulting from Mn-surface-modification using a facile oxidizing-coating method. Materials Letters, 2014, 115: 49-52.

[9] XIA SHU-BIAO, ZHANG YING-JIE, DONG PENG, et al. $\mathrm{CeO}_{2}$ surface modification to improve cycle and storage performance on lithium ion battery cathode material $\mathrm{LiNi}_{0.8} \mathrm{Co}_{0.15} \mathrm{Al}_{0.05} \mathrm{O}_{2}$. Chin. Journal of Inorganic Chemistry, 2014, 30(3): 529-535.

[10] JU S H, KANG I S, LEE Y S, et al. Improvement of the cycling performance of $\mathrm{LiNi}_{0.6} \mathrm{Co}_{0.2} \mathrm{Mn}_{0.2} \mathrm{O}_{2}$ cathode active materials by a dual-conductive polymer coating. ACS Applied Materials and Interfaces, 2014, 6(4): 2546-2552.

[11] LEE S H, YOON C S, KHALIL A, et al. Improvement of long-term cycling performance of $\mathrm{Li}\left(\mathrm{Ni}_{0.8} \mathrm{Co}_{0.15} \mathrm{Al}_{0.05}\right) \mathrm{O}_{2}$ by $\mathrm{AlF}_{3}$ coating . Journal of Power Sources, 2013, 234: 201-207.

[12] YANG H Z, LIU P X, CHEN Q L, et al. Fabrication and characteristics of high-capacity $\mathrm{LiNi}_{0.8} \mathrm{Co}_{0.15} \mathrm{Al}_{0.05} \mathrm{O}_{2}$ with monodisperse yolk-shell spherical precursors by a facile method . RSC Advances, 2014, 4: 35522-35527.

[13] WU N T, WU H, YUAN W, et al. Facile synthesis of one-dimensional $\mathrm{LiNi}_{0.8} \mathrm{Co}_{0.15} \mathrm{Al}_{0.05} \mathrm{O}_{2}$ microrods as advanced cathode materials for lithium ion batteries. Journal of Materials Chemistry A, 2015, 3: 13648-13652.

[14] YE NAI-QING, LIU CHANG-JIU, SHEN SHANG-YUE. Drawbacks and improve ways of $\mathrm{LINiO}_{2}$ as a cathode material for lithium ion batteries. Journal of Inorganic Materials, 2004, 19(6): $1217-1224$.

[15] LIN F, DENNIS N, LI Y Y, et al. Metal segregation in hierarchically structured cathode materials for high-energy lithium batteries. Nature Energy, 2016, 1: 15004.

[16] MAKIMURA Y, ZHENG S J, IKUHARA Y C, et al. Microstructural observation of $\mathrm{LiNi}_{0.8} \mathrm{Co}_{0.15} \mathrm{Al}_{0.05} \mathrm{O}_{2}$ after charge and discharge by scanning transmission electron microscopy. Journal of The Electrochemical Society, 2012, 159(7): A1070-A1073.

[17] CHO Y H, OH P, CHO J. A new type of protective surface layer for high-capacity Ni-based cathode materials: nanoscaled surface pillaring layer. Nano Letters, 2013, 13: 1145-1152.

[18] KIM H J, KIM M G, JEONG H Y, et al. A new coating method for alleviating surface degradation of $\mathrm{LiNi}_{0.6} \mathrm{Co}_{0.2} \mathrm{Mn}_{0.2} \mathrm{O}_{2}$ cathode material: nanoscale surface treatment of primary particles. Nano Letters, 2015, 15: 2111-2119.

[19] KONDO H, TAKEUCHI Y, SASAKI T, et al. Effects of $\mathrm{Mg}$-substitution in $\mathrm{Li}(\mathrm{Ni}, \mathrm{Co}, \mathrm{Al}) \mathrm{O}_{2}$ positive electrode materials on the crystal structure and battery performance. Journal of Power Sources. 2007, 174(2): 1131-1136.

[20] SATHIYAMOORTHI R, SHAKKTHIVEL P, RAMALAKSHMI S, et al. Influence of $\mathrm{Mg}$ doping on the performance of $\mathrm{LiNiO}_{2}$ matrix ceramic nanoparticles in high-voltage lithium-ion cells. Journal of
Power Sources. 2007, 171(2): 922-927.

[21] HUANG B, LI X, WANG Z, et al. Synthesis of Mg-doped Li$\mathrm{Ni}_{0.8} \mathrm{Co}_{0.15} \mathrm{Al}_{0.05} \mathrm{O}_{2}$ oxide and its electrochemical behavior in high-voltage lithium-ion batteries. Ceramics International, 2014, 40: $13223-13230$.

[22] POUILLERIE C, CROGUENNEC L, BIENSAN P, et al. Synthesis and characterization of new $\mathrm{LiNi}_{1-y} \mathrm{Mg}_{y} \mathrm{O}_{2}$ positive electrode materials for lithium-ion batteries. Journal of The Electrochemical Society, 2000, 147: 2061-2067.

[23] WU F, TIAN J, SU Y F, et al. Effect of $\mathrm{Ni}^{2+}$ content on lithium/nickel disorder for Ni-rich cathode materials. ACS Appl. Mater. Interfaces, 2015, 7: 7702-7708.

[24] CHEN Z Z, YUAN X J, WEN M S, et al. Preparation of Li$\mathrm{Ni}_{0.80} \mathrm{Co}_{0.15} \mathrm{Al}_{0.05} \mathrm{O}_{2}$ cathode material via Li-rich method. Energy Storage Science and Technology, 2014, 3(6): 620-623.

[25] BI Y J, YANG W C, DU R, et al. Correlation of oxygen non-stoichiometry to the instabilities and electrochemical performance of $\mathrm{LiNi}_{0.8} \mathrm{Co}_{0.1} \mathrm{Mn}_{0.1} \mathrm{O}_{2}$ utilized in lithium ion battery. Journal of Power Sources, 2015, 283: 211-218.

[26] LI X, XIE Z W, LIU W J, et al. Effects of fluorine doping on structure, surface chemistry, and electrochemical performance of $\mathrm{LiNi}_{0.8} \mathrm{Co}_{0.15} \mathrm{Al}_{0.05} \mathrm{O}_{2}$. Electrochimica Acta, 2015, 174: 1122-1130.

[27] ZHUANG G R V, CHEN G Y, SHIM J, et al. $\mathrm{Li}_{2} \mathrm{CO}_{3}$ in Li$\mathrm{Ni}_{0.8} \mathrm{Co}_{0.15} \mathrm{Al}_{0.05} \mathrm{O}_{2}$ cathodes and its effects on capacity and power. Journal of Power Sources, 2004, 134(2): 293-297.

[28] LIU W M, HU G R, DU K, et al. Enhanced storage property of $\mathrm{LiNi}_{0.8} \mathrm{Co}_{0.15} \mathrm{Al}_{0.05} \mathrm{O}_{2}$ coated with $\mathrm{LiCoO}_{2}$. Journal of Power Sources, 2013, 230: 201-206.

[29] ZHU L, LIU Y, WU W Y, et al. Surface fluorinated Li$\mathrm{Ni}_{0.8} \mathrm{Co}_{0.15} \mathrm{Al}_{0.05} \mathrm{O}_{2}$ as a positive electrode material for lithium ion batteries. Journal of Materials Chemistry A, 2015, 3: 15156-15162.

[30] XIONG X H, WANG Z X, YUE P, et al. Washing effects on electrochemical performance and storage characteristics of Li$\mathrm{Ni}_{0.8} \mathrm{Co}_{0.1} \mathrm{Mn}_{0.1} \mathrm{O}_{2}$ as cathode material for lithium-ion batteries. Journal of Power Sources, 2013, 222: 318-325.

[31] ZHENG X B, LI X H, WANG Z X, et al. Investigation and improvement on the electrochemical performance and storage characteristics of $\mathrm{LiNiO}_{2}$-based materials for lithium ion battery. Electrochimica Acta, 2016, 191: 832.

[32] BI Y J, WANG T, LIU M, et al. Stability of $\mathrm{Li}_{2} \mathrm{CO}_{3}$ in cathode of lithium ion battery and its influence on electrochemical performance. RSC Advances, 2016, 6: 19233-19237.

[33] DOKKO K, NISHIZAWA M, HORIKOSHI S, et al. In situ observation of $\mathrm{LiNiO}_{2}$ single-particle fracture during $\mathrm{Li}$-ion extraction and insertion. Electrochemical and Solid-State Letters, 2000, 3(3): $125-127$.

[34] ROBERT R, BÜNZLI C, BERG E J, et al. Activation mechanism 
of $\mathrm{LiNi}_{0.8} \mathrm{Co}_{0.15} \mathrm{Al}_{0.05} \mathrm{O}_{2}$ : surface and bulk operando electrochemical, differential electrochemical mass spectrometry, and $\mathrm{X}$ ray diffraction analyses. Chemistry of Materials, 2015, 27: 526-536.

[35] ITOU Y, UKYO Y. Performance of $\mathrm{LiNiCoO}_{2}$ materials for advanced lithium-ion batteries. Journal of Power Sources, 2005, 146(1/2): $39-44$

[36] ZHENG S J, HUANG R, MAKIMURA Y, et al. Microstructural changes in $\mathrm{LiNi}_{0.8} \mathrm{Co}_{0.15} \mathrm{Al}_{0.05} \mathrm{O}_{2}$ positive electrode material during the first cycle. Journal of The Electrochemical Society, 2011, 158(4): A357-A362.

[37] WATANABE S, KINOSHIRA M, HASOKAWA T, et al. Capacity fade of $\mathrm{LiAl}_{y} \mathrm{Ni}_{1-x-y} \mathrm{Co}_{x} \mathrm{O}_{2}$ cathode for lithium-ion batteries during accelerated calendar and cycle life tests (surface analysis of $\mathrm{LiAl}_{y} \mathrm{Ni}_{1-x-y} \mathrm{Co}_{x} \mathrm{O}_{2}$ cathode after cycle tests in restricted depth of discharge ranges). Journal of Power Sources, 2014, 258: 210-217.

[38] MAKIMURA Y, ZHENG S J, IKUHARA Y, et al. Microstructural observation of $\mathrm{LiNi}_{0.8} \mathrm{Co}_{0.15} \mathrm{Al}_{0.05} \mathrm{O}_{2}$ after charge and discharge by scanning transmission electron microscopy. Journal of The Electrochemical Society, 2012, 159(7): A1070-A1073.

[39] NOH H J, YOUN S, YOON C S, et al. Comparison of the structural and electrochemical properties of layered $\mathrm{Li}\left[\mathrm{Ni}_{x} \mathrm{Co}_{y} \mathrm{Mn}_{z}\right] \mathrm{O}_{2}$ $(x=1 / 3,0.5,0.6,0.7,0.8$ and 0.85$)$ cathode material for lithium-ion batteries. Journal of Power Sources, 2013, 233: 121-130.

[40] OHZUKU T, UEDA A, NAGAYAMA M. Electrochemistry and structural chemistry of $\mathrm{LiNiO}_{2}(\mathrm{R}-3 \mathrm{~m})$ for $4 \mathrm{~V}$ secondary lithium cells. Journal of The Electrochemical Society, 1993, 140: 1862-1870.

[41] YANG X Q, SUN X, MCBREEN J. Structural changes and thermal stability: In situ X-ray diffraction studies of a new cathode material $\mathrm{LiMg}_{0.125} \mathrm{Ti}_{0.125} \mathrm{Ni}_{0.75} \mathrm{O}_{2}$. Electrochemical Communications, 2000, 2: 733-737.

[42] SUN Y K, CHEN Z H, NOH H J, et al. Nanostructured high-energy cathode materials for advanced lithium batteries. $\mathrm{Na}$ ture Materials, 2012, 11: 942-947.

[43] LEE E J, CHEN Z H, NOH H J, et al. Development of microstrain in aged lithium transition metal oxides. Nano Letters, 2014, 14: 4873-4880.

[44] YANG J, XIA Y Y. Suppressing the phase transition of the layered Ni-rich oxide cathode during high-voltage cycling by introducing low-content $\mathrm{Li}_{2} \mathrm{MnO}_{3}$. ACS Applied Materials and Interfaces, 2016, 8(2): $1297-1308$.
[45] KOSTECKI R, MCLARNON F. Local-probe studies of degradation of composite $\mathrm{LiNi}_{0.8} \mathrm{Co}_{0.15} \mathrm{Al}_{0.05} \mathrm{O}_{2}$ cathodes in high-power Lithium-ion cells. Electrochemical and Solid-State Letters, 2004, 7(10): A380-A383.

[46] KOSTECKI R, MCLARNON F. Degradation of $\mathrm{LiNi}_{0.8} \mathrm{Co}_{0.2} \mathrm{O}_{2}$ cathode surfaces in high-power lithium-ion batteries. Electrochemical and Solid-State Letters, 2002, 5(7): A164-A166.

[47] KOSTECKI R, LEI J L, MCLARNON F, et al. Diagnostic evaluation of detrimental phenomena in high-power lithium-ion batteries. Journal of The Electrochemical Society, 2006, 153(4): A669-A672.

[48] LEI J L, MCLARNON F, KOSTECKI R, et al. In situ Raman microscopy of individual $\mathrm{LiNi}_{0.8} \mathrm{Co}_{0.15} \mathrm{Al}_{0.05} \mathrm{O}_{2}$ particles in a $\mathrm{Li}$-ion battery composite cathode. Journal of Physical Chemistry B, 2005, 109(2): 952-957.

[49] SASAKI T, NONAKA T, OKA H, et al. Capacity-fading mechanisms of $\mathrm{LiNiO}_{2}$-based lithium-ion batteries I. Analysis by electrochemical and spectroscopic examination. Journal of The Electrochemical Society, 2009, 156(4): A289-A293.

[50] MUTO S, SASANO Y, TATSUMI K, et al. Capacity-fading mechanisms of $\mathrm{LiNiO}_{2}$-based lithium-ion batteries II. Diagnostic analysis by electron microscopy and spectroscopy. Journal of The Electrochemical Society, 2009, 156(5): A371-A377.

[51] KOJIMA Y, MUTO S, TATSUMI K, et al. Degradation analysis of a Ni-based layered positive-electrode active material cycled at elevated temperatures studied by scanning transmission electron microscopy and electron energy-loss spectroscopy. Journal of Power Sources, 2011, 196: 7721-7727.

[52] BAK S M, NAM K W, CHANG W Y, et al. Correlating structural changes and gas evolution during the thermal decomposition of charged $\mathrm{Li}_{x} \mathrm{Ni}_{0.8} \mathrm{Co}_{0.15} \mathrm{Al}_{0.05} \mathrm{O}_{2}$ cathode materials. Chemistry of Materials, 2013, 25: 337-351.

[53] WU L J, NAM K W, WANG X J, et al. Structural origin of overcharge-induced thermal instability of Ni-containing layered-cathodes for high-energy-density lithium batteries. Chemistry of Materials, 2011, 23: 3953-3960.

[54] SHIM J, KOSTECKI R, RICHARDSON T, et al. Electrochemical analysis for cycle performance and capacity fading of a lithium-ion battery cycled at elevated temperature. Journal of Power Sources, 2002, 112: 222-230. 